\title{
Pharmacological BACE Inhibition Improves Axonal Regeneration in Nerve Injury and Disease Models
}

\author{
Carolyn Tallon ${ }^{1} \cdot$ Katherine L. Marshall ${ }^{1} \cdot$ Matthew E. Kennedy ${ }^{2} \cdot$ Lynn A. Hyde $^{3} \cdot$ Mohamed. H. Farah $^{1}$
}

Published online: 31 March 2020

(C) The American Society for Experimental NeuroTherapeutics, Inc. 2020

\begin{abstract}
While the peripheral nervous system is able to repair itself following injury and disease, recovery is often slow and incomplete, with no available treatments to enhance the effectiveness of regeneration. Using knock-out and transgenic overexpressor mice, we previously reported that BACE1, an aspartyl protease, as reported by Hemming et al. (PLoS One 4:12, 2009), negatively regulates peripheral nerve regeneration. Here, we investigated whether pharmacological inhibition of BACE may enhance peripheral nerve repair following traumatic nerve injury or neurodegenerative disease. BACE inhibitor-treated mice had increased numbers of regenerating axons and enhanced functional recovery after a sciatic nerve crush while inhibition increased axonal sprouting following a partial nerve injury. In the SOD1 ${ }^{\text {G93A }}$ ALS mouse model, BACE inhibition increased axonal regeneration with improved muscle re-innervation. CHL1, a BACE1 substrate, was elevated in treated mice and may mediate enhanced regeneration. Our data demonstrates that pharmacological BACE inhibition accelerates peripheral axon regeneration after varied nerve injuries and could be used as a potential therapy.
\end{abstract}

Key Words Peripheral nerve repair $\cdot$ BACE1 $\cdot$ peripheral nerve injury $\cdot$ amyotrophic lateral sclerosis $\cdot$ neurodegeneration.

\section{Introduction}

A fascinating aspect of the peripheral nervous system is its ability to regenerate axons following injury or disease, a feat not readily performed by the central nervous system. However, this regeneration is often unsatisfactory due to the slow and inefficient nature of the regenerative process. Despite the advances in understanding nerve regeneration [1-4], there are no therapeutic options available to patients that enhance regeneration and improve recovery. Levels of $\beta$-site amyloid precursor protein (APP)-cleaving enzyme 1 (BACE1) have been shown to regulate peripheral nerve

Electronic supplementary material The online version of this article (https://doi.org/10.1007/s13311-020-00852-3) contains supplementary material, which is available to authorized users.

Mohamed. H. Farah

mfarah2@jhmi.edu

1 Department of Neurology, Neuromuscular Division, Johns Hopkins University School of Medicine, The John G. Rangos Sr. Building, Room 239, 855 N. Wolfe Street, Baltimore, MD 21205, USA

2 Merck \& Co, Boston, MA, USA

3 Merck \& Co, Kenilworth, NJ, USA regeneration. BACE1 is a transmembrane protease involved in cleaving over 60 substrates as discussed by Hemming et al. [5-13], including APP and neuregulin-1 type III, which controls Schwann cell-mediated myelination of peripheral nerves $[9,10,14,15]$. Previously, we have demonstrated that genetic deletion of BACE1 leads to improved nerve regeneration [16], while the inverse scenario of overexpressing BACE1 leads to worsened regenerative outcomes in mice [17]. Additionally, others have shown that multiple BACE1 substrates also influence neurite outgrowth $[18,19]$. When the cleavage of NCAM and CHL1 is prevented, neurite outgrowth is enhanced [20, 21]. Thus, BACE1 activity levels inversely influence peripheral nerve regeneration, suggesting that pharmacologically reducing BACE1 activity could lead to enhanced peripheral nerve regeneration following axonal injury resulting from trauma or disease.

Potent and selective inhibitors of BACE that are welltolerated by patients have been developed for Alzheimer's Disease (AD), including the BACE1 inhibitor developed by Merck (Boston, MA) that we use in this study [22, 23]. We set out to investigate the ability of this BACE1 inhibitor to enhance regeneration in models of traumatic nerve injury and neurodegenerative disease. To model traumatic nerve injury, we used both a complete sciatic nerve crush and a partial cut 
injury of the branches of the lateral thoracic nerve (LTN) [24]. We then used a well-characterized model of motor neuron disease, the SOD1 ${ }^{\mathrm{G} 93 \mathrm{~A}}$ mouse model of amyotrophic lateral sclerosis (ALS) [25], to examine the impact of BACE1 inhibition in neurodegenerative disease. Mice which express the G93A mutant form of superoxide dismutase 1 (SOD1) exhibit early denervation of neuromuscular junctions well before motor neuron death [25-31]. We hypothesized that treating these mice with BACE inhibitors early on may encourage surviving axons to sprout and re-innervate muscle regions, potentially prolonging motor function.

The data presented in this paper support a use for BACE1 inhibitors as a novel therapy for enhancing peripheral nerve regeneration both following acute nerve injuries as well as in the context of motor neuron disease.

\section{Materials and Methods}

Study Design This study was conducted in order to identify clinically relevant BACE inhibitors as a potential therapy for enhancing peripheral nerve regeneration in the context of both peripheral nerve injury and early stage motor neuron disease. We used WT and SOD $1^{\mathrm{G} 93 \mathrm{~A}}$ mice, both on the C57BL/6 background, over the course of this study and all experiments were approved by the Animal Care and Use Committee of the Johns Hopkins University School of Medicine. The time points used in this study were decided on based on our previous experience and knowledge of the time course of peripheral nerve regeneration. Sample sizes were decided upon based on previous power calculations and our previous experience conducting such experiments [16, 17, 31]. Equal numbers of mice were randomly assigned a number and sorted into either a control vehicle group or an inhibitor treatment group. Not all the mice initially enrolled survived until end point analysis and therefore not all the studies had equal numbers of mice at the time of analysis. While sex differences have been reported for the onset of clinical symptoms and survival [32], previously our group did not observe sex differences in early denervation of asymptomatic mice [31]. In this study, again, we did not observe significant differences in the early denervation between male and female mice, and therefore, the data was pooled together according to genotype and treatment group. At the time of data collection, the treatment status of the mice was unknown and only after all the data was collected was the treatment conditions revealed in order to carry out statistical analysis.

Drug Information MBi-9 is a potent inhibitor of human BACE1 $(\mathrm{Ki}=1.5 \mathrm{nM})$ and human BACE2 $(\mathrm{Ki}=2.3 \mathrm{nM})$ enzymes in vitro and in cells (HEK293-APP ${ }^{\text {swe/Lon }}$ Ab40 IC50 $=2.2 \mathrm{nM}$ ) and displays $>10,000$-fold selectivity against multiple human aspartyl proteases. MBi- 9 was formulated in diet to produce sustained $>75 \%$ inhibition of CNS BACE1 activity. MBi- 9 was incorporated into the diet by Research Diets (New Brunswick, NJ). The structure and full pharmacological profile of MBi- 9 will be published at a later date. MBi9 was generously provided by Merck \& Co. LY2886721 was originally developed by Eli Lilly [33] is now commercially available and was purchased from DC Chemicals (Shanghai, China).

In Vitro Experiments Primary DRG cultures were isolated from 3-month-old C57B1/6 mice (Jackson Laboratories, Bar Harbor, MN) as previously described. DRGs were dissected from 4 mice and placed into ice-cold Leibovitz's L-15 Medium + 1 X penicillin/streptomycin (Thermo Fisher Scientific, Waltham, MA) and mechanically teased apart to assist with dissociation. DRGs were then placed in $0.18 \%$ trypsin media and further digested by pipetting up and down to dissociate cells. Cells were then spun down at $1000 \mathrm{rpm}$ for $1 \mathrm{~min}$. The pellet was resuspended in $10 \mathrm{mg} / \mathrm{ml}$ collagenase $+\mathrm{L}-15$ medium and incubated at $37^{\circ} \mathrm{C}$ for $45 \mathrm{~min}$ at which point cells were further dissociated by slowly pipetting several times before being spun at $1000 \mathrm{rpm}$ for $1 \mathrm{~min}$. The pellet was resuspended in $0.18 \%$ trypsin + $0.5 \mathrm{mg} / \mathrm{ml}$ DNase + L- 15 medium and the reaction was stopped after $15 \mathrm{~min}$ by adding growth medium (1X Penn/ Strep + 1X N2 medium+10\% FBS in DMEM/F12 medium, all from Thermo Fisher Scientific) before being spun down at $1000 \mathrm{rpm}$ for $2 \mathrm{~min}$ and washing the pellet 2 times. After the final wash, the pellet was resuspended in $0.5 \mathrm{mg} / \mathrm{ml}$ DNase solution and then passed through a $0.22 \mu \mathrm{m}$ filter where L-15 medium was added afterwards and spun down at $1000 \mathrm{rpm}$ for $10 \mathrm{~min}$. The final pellet was resuspended in growth medium and cells were plated on laminin and poly-D lysine (Millipore Sigma, Burlington, MA) coated glass coverslips in a 24-well dish at a density of $5 \times 10^{4}$ cells/well. The cells were treated with either vehicle $(0.0002 \%$ DMSO in growth medium) or LY2886721 (100 nM) and allowed to grow for 2 days. Cells were there fixed with $2 \%$ paraformaldehyde for $10 \mathrm{~min}$ before being blocked for $1 \mathrm{~h}$ in $5 \%$ goat serum (Jackson Laboratories) $+0.1 \%$ Triton X-100 (Sigma-Aldrich) and room temperature then stained overnight at $4{ }^{\circ} \mathrm{C}$ with a $\beta$-tubulin III primary antibody (Millipore Sigma). Cells were then stained with an appropriate secondary antibody and imaged using a Zeiss AxioVert microscope with an apotome attachment (Zeiss, Oberkochen, Germany). The number of branches/soma, length of the longest neurite, and the number of branches/longest neurite were all quantified using ImageJ software.

Pharmacokinetic Analysis 2-month old SOD1 mice were split into 4 different groups and given either $1,3,10$, or $30 \mathrm{mg} / \mathrm{kg}$ doses of MBi- 9 chow ( $n=3$ /group) and WT mice were given $30 \mathrm{mg} / \mathrm{kg}$ of MBi- 9 chow $(n=3)$. Mice were singly housed 
and treated for 10 days. The amount of food eaten per day and the daily body weight was measured throughout the dosing period. Plasma and brain concentrations were determined as previously described $[34,35]$. After 10 days of treatment, mice were deeply anesthetized with $10 \%$ chloral hydrate (Sigma-Aldrich, St. Louis, MO) and their blood was collected via cardia puncture into heparin-coated tubes (Thermo Fisher Scientific). The blood was spun down at $3000 \mathrm{rpm}$ for $15 \mathrm{~min}$ and the plasma was collected and frozen on dry ice before being stored long term at $-80{ }^{\circ} \mathrm{C}$. The mice were then perfused with ice-cold 1X PBS before dissecting out the whole brains which were then snap frozen in liquid nitrogen and stored at $-80^{\circ} \mathrm{C}$. The actual dose calculated from the amount of chow being eaten was done by multiplying the dose in the chow by the amount of drug eaten then dividing by the body weight of the mouse (diet dose*drug eaten/body weight). The amount of drug measured in the plasma and brain samples was determined by liquid chromatography coupled with tandem mass spectrometry (LC-MS/MS) using non-validated assays.

BACE1 KO Experiments A total of 4 WT and 4 BACE1 KO mice were used in this study. All breeding mice were obtained from Jackson Laboratories. Yellow fluorescent protein (YFP)transgenic mice (B6.Cg-Tg(Thy1-YFP)16Jrs/J), in which all the motor axons express YFP fluorescence, [36] were bred with BACE1 KO mice crossed 10 times to the B6 background, that were generously donated by Dr. Alena Savonenko at Johns Hopkins University, to generate KO mice expressing YFP in all their axons. Mice were 3-4 months old at the time of experiments.

Nerve Injury Experiments WT type mice on the B6 background were obtained from existing breeding cages originally obtained from Jackson Laboratories. For the inhibitor studies following a sciatic nerve crush, a total of 53 mice were used, with 29 on the vehicle and 24 on the inhibitor treatment. For the partial nerve transection studies, WT mice expressing YFP on the B6 background from Jackson Laboratories were used (B6.Cg-Tg(Thy1-YFP)16Jrs/J). A total of 10 mice were used, with 3 on the vehicle and 4 on the inhibitor treatment. 3 YFP mice were used to determine the reliability of a partial nerve transection in the LTN-CMM system by carrying out the partial nerve transections and comparing the qualitative extent of denervation observed. The backs of these mice were collected and imaged to determine whether there were extensive areas of partial denervation in order to maximize the likelihood of axonal sprouting. All 3 of the mice demonstrated robust areas of partial and complete denervation bordering areas of innervation, therefore, we felt confident in our model.

SOD1 Experiments All breeder mice were obtained from Jackson Laboratories. Yellow fluorescent protein (YFP)-transgenic mice (B6.Cg-Tg(Thy1-YFP)16Jrs/J) that express YFP in all motor neurons [36] were bred with SOD1 transgenic mice (B6.Cg- $\mathrm{Tg}(\mathrm{SOD} 1 * \mathrm{G} 93 \mathrm{~A}) 1 \mathrm{Gur} / \mathrm{J})$ [37], which exhibit an ALS-like phenotype and express a G93A mutant form of human SOD1. Both of these lines were in the C57BL/6J genetic background. Progeny were genotyped and grouped into a SOD $1{ }^{\mathrm{G} 93 \mathrm{~A}}$ carrying transgenic group (SOD1) and wild type littermates (WT). Over the entire course of the studies, the SOD1 mice were absent of any muscle weakness or disease phenotype and did not exhibit any weight loss. A total of 142 SOD1 mice were used, with 69 on vehicle and 73 on inhibitor treatment. Mice were between 2 and 3 months of age at the time of the experiments. Control littermates were designated as WT.

LTN-CMM Electrophysiology The protocol followed was the same as previously described in Tallon et al. [31]. Briefly, mice were anesthetized using 2-3\% isoflurane (Baxter, San Juan, Puerto Rico) and the hair on the back of the trunk was shaved with electric clippers. Using an Evidence 3102evo EMG system (Schreiber \& Tholen Medizintechnik, Stade, Germany), compound muscle action potentials (CMAPs) were recorded from 3 sites along the CMM and were designated caudal (region closest to the tail), medial and rostral (region closest to the head). The LTN was stimulated for $0.05 \mathrm{~ms}$ at $1 \mathrm{~Hz}$ by making an incision just below the left shoulder blade to expose the LTN and hooking the middle branch of LTN with the electrode. The maximal CMAPs from each recording position, starting at the caudal site, were then recorded and the amplitudes and latencies were used to analyze neuromuscular function.

Sciatic Nerve Electrophysiological Experiments A standard sciatic nerve electrophysiology protocol was followed using an Evidence 3102evo EMG system and as previously described $[17,31]$. Briefly, a very short, supramaximal electrical impulse $(<0.2 \mathrm{~ms}$ at $10-15 \mathrm{mV})$ was used to electrically stimulate the left sciatic nerve via stimulating electrodes placed at the ankle in anesthetized mice. The CMAPs generated were recorded by inserting recording electrodes into the plantar muscle of the foot. The distance between the stimulating and recording sites was approximately $12 \mathrm{~mm}$. The amplitude, latency, and area under the curve were analyzed as measures of sciatic nerve regeneration following injury to the sciatic nerve.

Mouse Perfusion After the course of treatment was completed, the mice were anesthetized with isoflurane and the hair from the dorsal portion of the trunk of the body was shaved off with electric clippers. We then performed electrophysiological analysis of the LTN and sciatic nerve function. Immediately following the recordings, $10 \%$ chloral hydrate (SigmaAldrich) was used to deeply anesthetize the animals and an over -the -counter chemical depilatory was used to remove 
any remaining hair. Mice were first transcardially perfused with 1X PBS (Life Technologies, Carlsbad, CA) followed by a $2 \%$ paraformaldehyde (PFA) (Electron Microscopy Sciences, Hatfield, PA) in 1X PBS perfusion. The skin covering the dorsal portion of the trunk was removed with the CMM attached and then postfixed in $2 \%$ PFA for up to $16 \mathrm{~h}$ at room temperature before being transferred to PBS for long term storage at $4{ }^{\circ} \mathrm{C}$. The lower portion of the body was also postfixed in 2\% PFA overnight at room temperature before being transferred to PBS and stored at $4{ }^{\circ} \mathrm{C} .2 \%$ PFA was used to reduce background fluorescence at the YFP wavelength for imaging studies.

Western Blot Freshly harvested sciatic nerves and brains were flash frozen and stored at $-80^{\circ} \mathrm{C}$ before extracting protein by using a glass micro-homogenizer to homogenize tissue in extraction buffer (T-PER ${ }^{\circledR}$ tissue protein extraction reagent, Thermo Fisher Scientific) containing a protease inhibitor (Thermo Fischer Scientific). A Micro BCA ${ }^{\mathrm{TM}}$ Protein Assay Kit was used to determine protein concentration levels as per the manufacturer's instructions (Thermo Fisher Scientific). Protein was loaded onto a Novex WedgeWell 8-16\% TrisGlycine gel or a 4-20\% Tris-Glycine gel (both from Invitrogen, CA, USA) before transferring to a nitrocellulose membrane (Invitrogen). The blots were then blocked with 5\% milk (Bio-Rad, CA) or 5\% donkey serum (Millipore Sigma) and stained overnight at $4{ }^{\circ} \mathrm{C}$ with an anti-BACE1 [38], antiAPP (Millipore Sigma). The blots were then washed 3 times for $5 \mathrm{~min}$ in TBST before adding appropriate HRP conjugated secondary antibodies for $1 \mathrm{~h}$ at room temperature. The blots were washed again and then visualized on film using Amersham ECL detection reagent (GE Healthcare, UK). The blots were cut in half at an appropriate size in order to visualize loading controls using an anti-GAPDH-HRP conjugated antibody (Sigma-Aldrich) for $1 \mathrm{~h}$ at room temperature before being visualized in the same way as the other antibodies.

Sciatic Nerve Regeneration Analysis As quantification studies require smaller groups of mice, we randomly selected a subset of mice from the electrophysiology studies to utilize for regeneration quantification. For semi-thin sections, sciatic nerves were postfixed in a $2 \%$ paraformaldehyde/ $2 \%$ glutaraldehyde (Millipore Sigma) in 1X PBS mixture overnight at room temperature. They were then placed in $\mathrm{OsO} 4$ and embedded in Epon (all from Electron Microscopy Sciences). 0.5 - $\mu \mathrm{m}$-thick cross-sections were obtained and stained for toluidine blue for examination under light microscopy. In order to count all of the regenerated axons, $63 \mathrm{X}$ oilimmersion bright field images were tiled to obtain the complete area of the nerves using as Axio Imager fluorescence microscope (Zeiss). The number of myelinated regenerating axons were counted over the entire area of each nerve. The process for ultra-structural analysis was described previously [16]. Briefly, sciatic nerves were sectioned at $70 \mathrm{~nm}$ and stained for citrate/uranyl acetate. Electron micrographs were acquired using a Zeiss Libra transmission electron microscope.

Gastrocnemius Re-innervation As large numbers of NMJs can be quantified from fewer mice, we selected a smaller subset of animals from our electrophysiology studies for gastrocnemius quantification. Following $2 \%$ paraformaldehyde overnight fixation and storage in $1 \mathrm{X}$ PBS, the gastrocnemius muscle was dissected out and cryoprotected in $30 \%$ sucrose (Thermo Fisher Scientific) in $1 \times$ PBS overnight. The gastrocnemius muscles were then frozen onto a microtome stage before cutting 50- $\mu \mathrm{m}$-thick sections (Microm MH450, Thermo Fisher Scientific). Tissue sections were permeabilized and blocked by $5 \%$ goat serum (Jackson laboratories) $/ 0.3 \%$ Triton X-100 (Sigma-Aldrich) in $1 \times$ PBS for $1 \mathrm{~h}$ at room temperature. The sections were then stained overnight at $4{ }^{\circ} \mathrm{C}$ with primary antibodies against neurofilament (NF) and SV2 (Developmental Studies Hybridoma Bank, Iowa City, Iowa) to visualize the axons. $\alpha$-bungarotoxin conjugated to Alexa Fluor 647 ( $\alpha$-BTX, Life Technologies) and the corresponding Alexa Fluor 488 conjugated secondary antibodies (Life Technologies) were stained for $1 \mathrm{~h}$ at room temperature before being mounted in prolong diamond mounting reagent (Life Technologies) and cover-slipped. The sections were then imaged using a Zeiss LSM 800 confocal microscope. NMJs were scored as being innervated by having at least half of the YFP presynaptic expression overlapping with the postsynaptic $\alpha$-BTX staining.

CMM Staining Protocol The CMM was cleared of as much connective tissue as possible before being permeabilized and blocked by $5 \%$ goat serum (Jackson laboratories) $/ 1 \%$ Triton X-100 (Sigma-Aldrich)/5\% DMSO (SigmaAldrich) in $1 \mathrm{X}$ PBS for $1.5 \mathrm{~h}$ at room temperature. Sections were then incubated for $3 \mathrm{~h}$ at room temperature with $\alpha$-BTX conjugated to Alexa Fluor 647 in the permeabilization solution and then washed extensively in $1 \mathrm{X}$ PBS before being mounted (Prolong Diamond, Life Technologies) and cover-slipped. Stained sections were then imaged using a Zeiss LSM 800 confocal microscope. Innervated NMJs were scored as a complete overlap of the presynaptic YFP expression with the post-synaptic $\alpha$-BTX while denervated was scored as no overlapping staining.

Statistics Each mouse was assigned a random number and the specific genotype and treatment condition of each mouse was unknown at the time of data collection and analysis for all experiments performed in order to ensure blinded analysis. All statistical analyses between 2 sets of data were performed using a two-tailed Student's $t$ test 
while a repeated-measures ANOVA with multiple comparisons was used for statistical analysis of data over time. Where the data failed to pass the normalization test, the Mann-Whitney Rank Sum Test was performed. All data analysis was performed using SigmaPlot software (Systat Software Inc., Chicago, IL) and any value of $p<0.05$ was scored as statistically significant. All graphed data are presented as mean \pm SEM.

\section{Results}

\section{BACE Inhibition Enhances Axonal Outgrowth In Vitro}

Our initial studies aimed to determine whether inhibiting BACE activity was able to enhance neurite outgrowth in vitro. Primary adult DRG neurons were treated with either DMSO only (vehicle treatment) or $100 \mathrm{nM}$ of the BACE inhibitor LY2886721 for 2 days. Following treatment, we observed an increase in the number of neurite branches extending from the cell soma, the length of the longest neurite, and the number of branches on the longest neurite (Supplementary Figure 1). We therefore concluded that BACE inhibition enhanced neurite outgrowth in vitro and began to investigate whether BACE inhibition could enhance nerve regeneration in vivo.

\section{In Vivo Bioavailability of BACE Inhibitors}

Going forward with the in vivo studies, we utilized Merck's BACE inhibitor, MBi-9, as clinical trials with Merck's inhibitors did not observe any serious adverse side effects in patients, $[22,39]$ such as liver toxicity, which was observed with some earlier BACE inhibitors, including LY2886721. [33] Additionally, MBi-9 has excellent specificity for BACE over other aspartyl proteases (Supplemental Table 1). We observed that a $30 \mathrm{mg} / \mathrm{kg}$ dose of MBi-9, which produces a $>90 \%$ reduction of CNS A $\beta 40$ levels in WT mice [22, 34, 35], was able to inhibit BACE activity in the mouse brain and spinal cord (Supplemental figure 2 A-B). There was a significant increase in the intensity of the full-length APP bands in the inhibitor-treated mice with a 1.6-fold increase in the brain and a 3.4-fold increase in the spinal cord (Supplemental figure 2B). Therefore, we concluded that MBi-9 inhibited BACE activity in vivo and proceeded to use the inhibitor in subsequent experiments.

To achieve facile and long-lasting BACE inhibition, we opted to deliver MBi-9 mixed with diet chow. We ensured that providing MBi-9 via the mouse chow was giving a reliable dose over time by monitoring body weight and food consumption. We observed consistent dosing over a 10-day period with all doses tested $(1 \mathrm{mg} / \mathrm{kg}, 3 \mathrm{mg} / \mathrm{kg}$, $10 \mathrm{mg} / \mathrm{kg}$, and $30 \mathrm{mg} / \mathrm{kg}$; Supplemental figure 2C).
Additionally, we performed pharmacokinetic analysis of the concentration of MBi- 9 in the plasma and brain after the 10-day dosing experiment and found detectable levels of the drug in all concentrations tested (Supplemental figure 2D). The observation that the highest dose tested, $30 \mathrm{mg} / \mathrm{kg}$, was well-tolerated, present in both the plasma and spinal cord, and caused increased full-length APP allowed us to confidently carry out the rest of the experiments with this dose.

\section{BACE Inhibitor Treatment Does Not Alter Normal, Uninjured Axon Morphology and Muscle Innervation}

Before performing the injury experiments, we first verified whether a 2-week treatment of $30 \mathrm{mg} / \mathrm{kg}$ of MBi-9 had any effects on normal axon morphology and electrophysiology. We did not observe any obvious differences between the extent of myelination nor the size and abundance of healthy axons (Supplemental figure 3A and B). There were no observed differences in the innervation of the uninjured gastrocnemius muscle as measured by the percentage of innervated NMJs (Supplemental figure $3 \mathrm{C}$-E). There were also no significant differences between the compound muscle action potential (CMAP) amplitudes or latencies (Supplemental figure $3 \mathrm{~F})$, consistent with there being no difference in the myelination status or the number of healthy axons present in the sciatic nerve. This demonstrated that BACE inhibition in the healthy mouse did not alter normal axon morphology, innervation, or nerve physiology and we proceeded to investigate BACE inhibition following nerve injuries.

We also determined whether 1 month of BACE inhibitor treatment had any effects on NMJ innervation and terminal axonal sprouting since abolishing action potentials using tetrodotoxin [40] or presynaptically blocking at the NMJ with botulinum toxin [41] led to enhanced axonal sprouting despite a lack of axonal denervation. 1-month-old WT mice expressing YFP in their axons were treated with either vehicle chow $(n=4)$ or $30 \mathrm{mg} / \mathrm{kg}$ BACE inhibitor chow $(n=3)$ for 1 month. To facilitate quantification without needing more complicated staining methods, only terminal axonal sprouts were quantified where NMJs that had 2+ axons innervating them were scored as a sprout. Inhibitor treated mice did not display any significant changes in the percentage of innervated NMJs (Supplemental figure 4C) nor did they have any significant increase in the percentage of multiply innervated NMJs indicative of terminal sprouts (Supplemental figure 4D). Both vehicle and inhibitor treated mice had less than $0.5 \%$ of their NMJs displaying terminal sprouting. This demonstrates that BACE inhibition does not appear to trigger compensatory axonal sprouting via blockade of action potentials or NMJ transmission. 

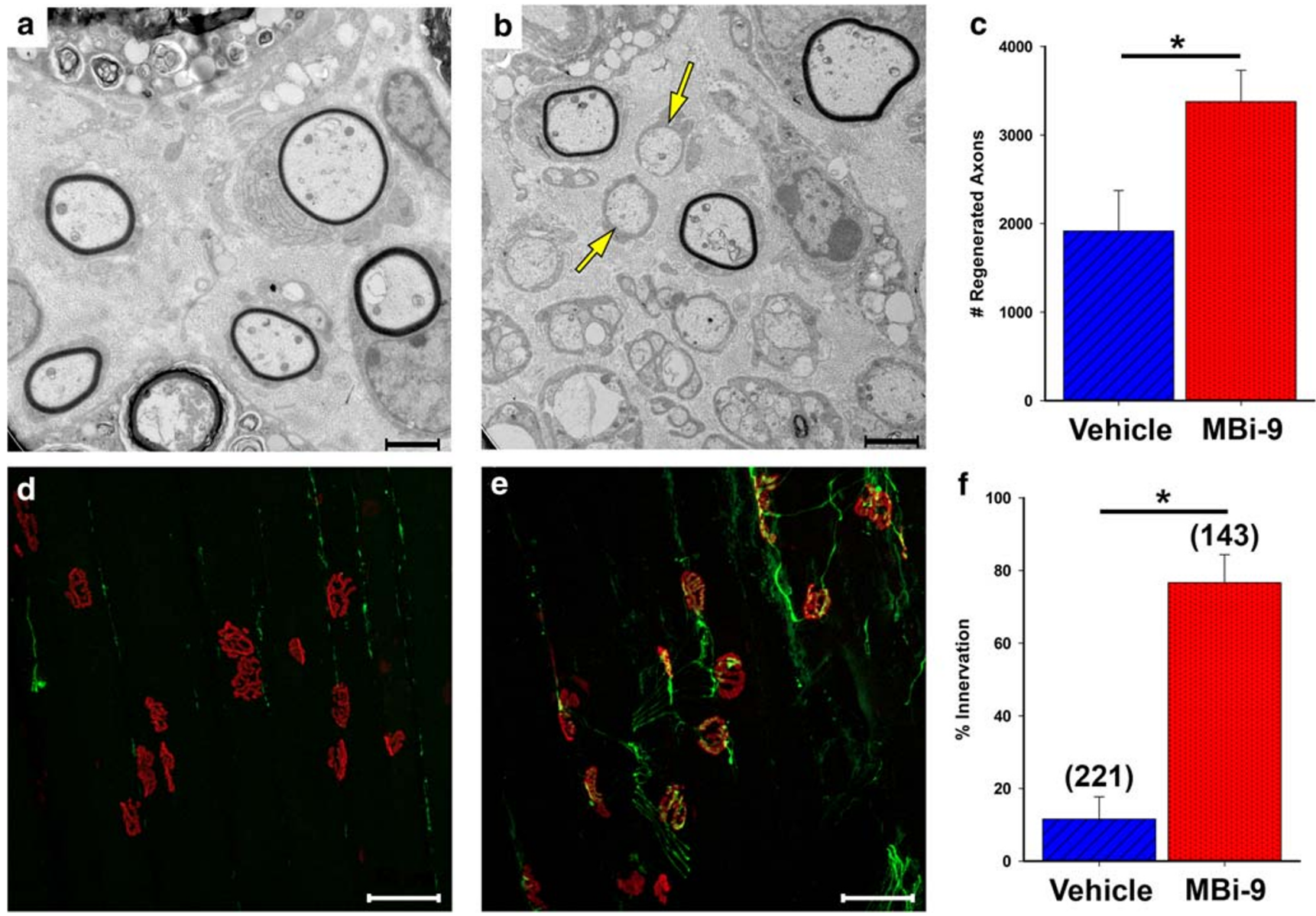

Fig. 1 BACE inhibitor MBi-9 enhances axonal regeneration following a sciatic nerve crush at 2 weeks after injury. (A, B) Representative EM images of sciatic nerve cross sections from vehicle (A) and inhibitor (B) treated mice showing many more regenerated axons in the treated images (yellow arrows). (C) Quantification of the number of regenerated axons in vehicle and inhibitor treated mice. Vehicle $n=4$, inhibitor $n=5$. (D, E) Representative images of sectioned gastrocnemius muscles from vehicle

\section{BACE Inhibition Enhances Axon Regeneration After a Sciatic Nerve Crush Injury}

We performed sciatic nerve crushes on C57BL/6J WT mice which received either vehicle $(n=4)$ or MBi-9 treatment (30 $\mathrm{mg} / \mathrm{kg} ; n=5$ ) for 2 weeks beginning at the time of nerve crush. Inhibitor-treated mice had many more regenerating axons in sciatic nerve cross-sections than those treated with vehicle (Fig. 1A, B). After quantification, we found that inhibitor-treated mice $(n=5)$ had significantly more regenerating axons than vehicle-treated mice $(n=4)$ ( $3378.40 \pm 353.78$ vs $1916.75 \pm 456.16$; $p=0.037$, Fig. 1C). We next determined whether these faster regenerating axons were reaching the gastrocnemius muscle and leading to improved NMJ re-innervation of the muscle. Inhibitor treated mice had significantly increased NMJ re-innervation (Fig. $1 \mathrm{D}-\mathrm{F})$. Inhibitor treated gastrocnemius muscles were $76.64 \% \pm 7.73$ re-innervated $(n=3,2$ mice had issues with
(D) and inhibitor (E) treated mice stained for SV2, NF (both green), and $\alpha$-BTX (red). (F) Quantification of the number of innervated NMJs between vehicle and inhibitor treated mice. Scale bars, $2 \mu \mathrm{m}(\mathbf{A}$ and $\mathbf{B})$ and $50 \mu \mathrm{m}$ (D and $\mathbf{E})$. Vehicle $\mathrm{n}=4$, inhibitor $n=3$. Numbers in parenthesis indicate total number of NMJs counted. Bars represent the mean \pm standard error. Statistical significance was calculated using a two-tailed Student's $t$ test. $* p<0.05$

proper tissue staining and so we decided to exclude them from the study) while vehicle-treated mice were only $11.55 \% \pm$ 6.12 re-innervated $(n=4)$ (Fig. 1F; $p=0.001$ ).

We next examined whether the improved regeneration induced by BACE inhibition translated to enhanced functional recovery after injury. We measured whether these increased numbers of re-innervated NMJs were able to elicit stronger compound muscle action potentials (CMAPs) in the plantar muscles of the foot pad to observe direct functional recovery of the sciatic nerve to the distal muscles it innervates. We crushed the left sciatic nerves of 21 mice and placed 12 on vehicle and 9 on inhibitor. We then followed the rate of recovery of the amplitude of the CMAP stimulated at the ankle site every 2 weeks for a total of 8 weeks (Fig. 2). 2 weeks following injury, neither the vehicle nor the inhibitor-treated mice had any significant recovery of the CMAP amplitude (Fig. 2I; vehicle $=0.045 \pm 0.017 \mathrm{mV}$; inhibitor $=0.038 \pm 0.021 ; p=$ 0.42). At 4 weeks, both the vehicle and inhibitor showed 

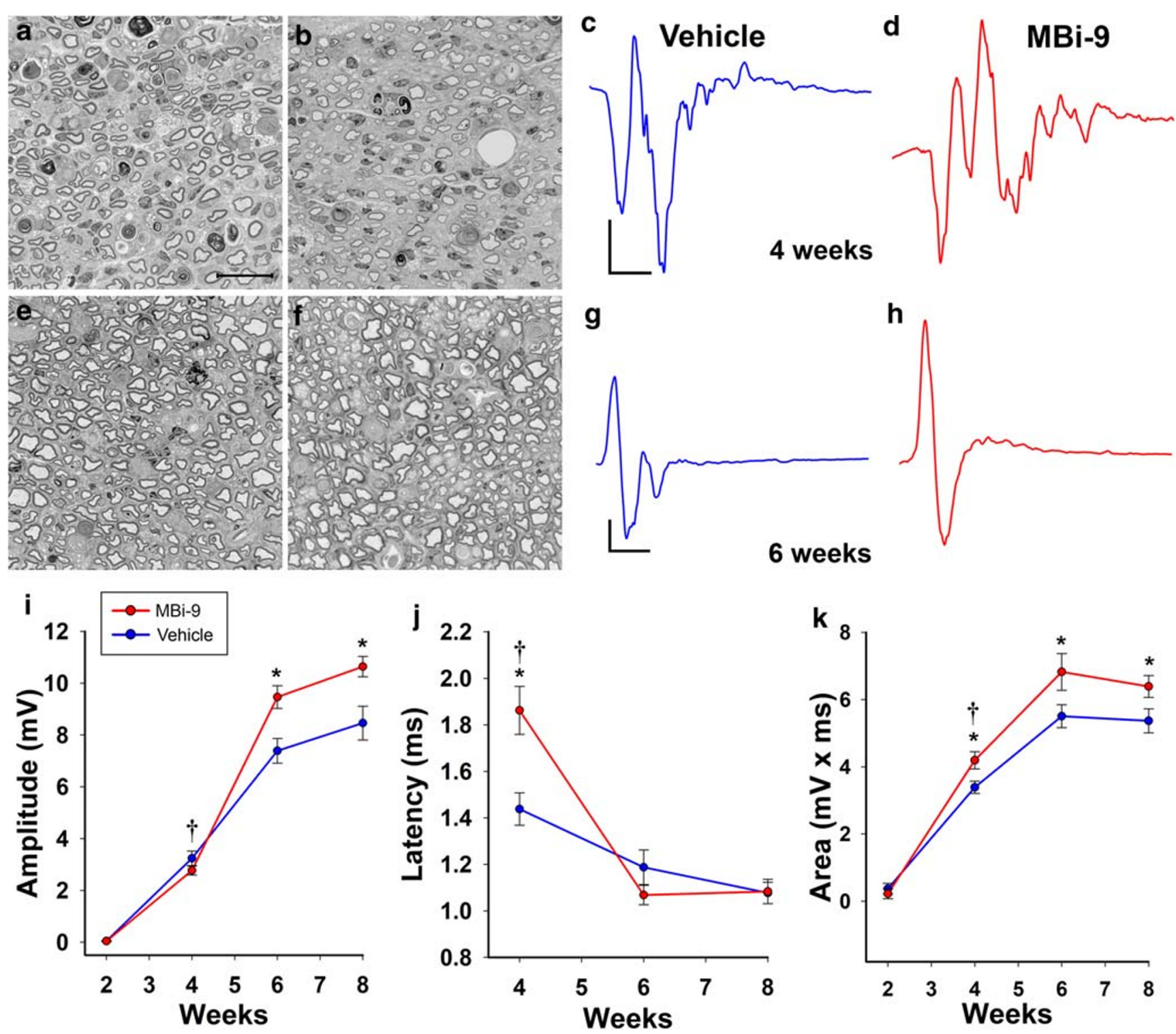

Fig. 2 MBi-9 treatment enhances functional repair following a sciatic nerve crush injury. (A, B) Representative images of Toluidine blue stained sciatic nerve cross sections from vehicle (A) and inhibitor (B) treated mice at 4 weeks after injury. (C, D) Representative CMAP traces at 4 weeks after injury from vehicle $(\mathbf{C})$ and inhibitor $(\mathbf{D})$ treated mice. (E, F) Representative images of Toluidine blue stained sciatic nerve cross sections from vehicle $(\mathbf{E})$ and inhibitor $(\mathbf{F})$ treated mice at 6 weeks after injury. (G, H) Representative traces at 6 weeks post injury from vehicle $(\mathbf{G})$ and inhibitor (H) treated mice. (I) Quantification of the

CMAP amplitudes recorded every 2 weeks over 8 weeks total. (J) Quantification of the latencies recorded every other week beginning at week 4. (K) Quantification of the area under the curve every 2 weeks for 8 weeks. Scale bars, $20 \mu \mathrm{m}(\mathbf{A}, \mathbf{B}, \mathbf{E}, \mathbf{F}) ; 2 \mathrm{~ms}(x)$ by $0.4 \mathrm{mV}(y)(\mathbf{C}, \mathbf{D})$; and $2 \mathrm{~ms}(x)$ by $1 \mathrm{mV}(y)(\mathbf{G}, \mathbf{H})$. Points represent the mean \pm standard error. Statistical significance was calculated using a repeated measure ANOVA with multiple comparisons. $* p<0.05$. The dagger denotes time point when BACE inhibitor treatment was halted 4 weeks after injury

similar improvements in the CMAP amplitude (Fig. 2I; vehicle $=3.24 \pm 0.28 \mathrm{mV}$; inhibitor $=2.77 \pm 0.17 \mathrm{mV} ; p=0.41$ ). However, we observed a significant delay in the latencies of the inhibitor treated mice $(1.86 \pm 0.10 \mathrm{~ms})$ compared with vehicle treated mice $(1.44 \pm 0.070 \mathrm{~ms})$ (Fig. $2 \mathrm{~J} ; p=0.0070)$, indicative of potential issues surrounding re-myelination. When we examined the morphology of the axons after 4 weeks, we observed thinner myelin in inhibitor-treated mice compared to vehicle-treated mice (Fig. 2A, B). Intriguingly, the shape of the inhibitor-treated CMAP traces had many more peaks

distributed throughout the duration of the CMAP compared to the traces from vehicle-treated animals (Fig. 2C, D). When we calculated the area under the curve of the traces, we found that inhibitor-treated mice had a significantly higher area than vehicle-treated mice (Fig. $2 \mathrm{~K}$; vehicle $=3.39 \pm 0.18 \mathrm{mV} \times \mathrm{ms}$; inhibitor $=4.20 \pm 0.26 \mathrm{mV} \times \mathrm{ms} ; p=0.015$ ). Together, this suggested that inhibitor-treated mice had a greater number of axons firing than vehicle-treated mice, however, these axons were not able to coherently fire together to generate a higher CMAP amplitude. This result likely reflects a defect in myelin 
thickness that is related to inhibiting BACE1's processing of neuregulin 1 type III and reduced re-myelination of the peripheral nerves as seen in BACE1 KO mice [9, 10, 42, 43]. Therefore, we decided to halt treatment after 4 weeks to allow for re-myelination to occur and test if an improvement in the CMAP amplitude could be observed in the inhibitor-treated mice. After 2 weeks off the inhibitor, 6 weeks following injury, the extent of myelination on the axons appeared similar and the traces of both the vehicle and inhibitor-treated mice were much more cohesive in their firing (Fig. 2E-H). The CMAP amplitudes of the inhibitor-treated mice that were washed out of BACE inhibitor for 2 weeks were significantly higher than the vehicle treated mice (Fig. 2I; vehicle $=7.39 \pm 0.48 \mathrm{mV}$; inhibitor $=9.47 \pm 0.44 \mathrm{mV} ; p=0.0031)$. At this time point, the latencies were no longer significantly different from one another (Fig. 2J; vehicle $=1.19 \pm 0.075 \mathrm{~ms}$; inhibitor $=1.07 \pm$ $0.042 ; p=0.16$ ), suggesting that re-myelination of the regenerating axons had occurred. We observed a similar trend at 8 weeks after injury, following 4 weeks without inhibitor, where the inhibitor treated amplitudes were significantly greater than the vehicle treated amplitudes (Fig. 2I; vehicle $=$ $8.46 \pm 0.65 \mathrm{mV}$; inhibitor $=10.64 \pm 0.39 ; p=0.0030)$. Again, the latencies were not significantly different (Fig. 2J; vehicle $=$ $1.08 \pm 0.046 \mathrm{~ms}$; inhibitor $=1.08 \pm 0.053 ; p=0.92$ ). Taken together, our data (Figs. 1 and 2) suggest that BACE inhibitor treatment enhances sciatic nerve regeneration and muscle reinnervation, as well as improves CMAP amplitude recovery following a complete sciatic nerve crush injury.

\section{Reduced BACE Activity Increases Axonal Sprouting Following a Partial Nerve Injury}

In order to study BACE inhibition's effect on collateral axonal sprouting from intact axons, we carried out a partial nerve injury experiment with a purely motor nerve, the lateral thoracic nerve (LTN), and the muscle it innervates, the back spanning cutaneous maximus muscle (CMM) [3, 24, 31]. We reasoned that this approach would more accurately model most traumatic nerve injury cases. We were able to reliably perform partial nerve transections by isolating the branches coming from the C6-C7 spinal cord regions near the shoulder and severing 2 of the 3 branches which led to large regions of partial innervation in 3 WT mice expressing YFP in their axons (Supplemental Fig. 5A-C).

We investigated whether pharmacological inhibition of BACE activity in adult mice enhances axonal sprouting following a partial nerve transection of the LTN of WT mice treated with either vehicle $(n=3)$ or MBi-9 inhibitor $(n=4)$. The mice were treated for 14 days before collecting the CMM to measure the extent of terminal axonal sprouting. To maximize the likelihood of locating axonal sprouts, images were taken from innervated or partially innervated regions directly adjacent to regions of denervation since axons will only sprout to nearby areas of denervation. For simplicity, only terminal axonal sprouts were scored as those are the easiest to define since healthy NMJs only have one axonal input. Any additional axonal outputs, either towards another NMJ or empty space, were scored as a terminal axonal sprout. We observed an increase in the presence of thin, extra-terminal axonal sprouts in mice treated with $\mathrm{MBi}-9$ when compared with the vehicle-treated mice (Fig. 3A, B; white arrows). After quantifying the number of terminal axonal sprouts in vehicle and inhibitor treated mice, there was a significant increase in the percentage of NMJs that had terminal axonal sprouts in the inhibitor treated mice when compared with vehicle treated mice (Fig. 3C; vehicle $=14.32 \pm 2.76 \%, n=262$ NMJs; inhibitor $=37.42 \pm 2.24 \%, n=311$ NMJs; $p=0.0012)$. These sprouting values are much greater than inhibitor-treated uninjured muscles, suggesting there may be an increase in the activation of injury response mechanisms $(<0.5 \%$, Supplemental Fig. 4). Additionally, we observed an increase in the number of NMJs that had at least one terminal axonal sprout in BACE1 KO mice versus WT control mice (Supplemental Figure 6 A-C). This data demonstrates that reducing the activity of $\mathrm{BACE} 1$, either genetically (Supplemental Fig. 6) or pharmacologically (Fig. 3), results in increased axonal sprouting following a partial nerve injury.

\section{BACE Inhibition Improves Muscle Innervation and Electrophysiological Profiles in an ALS Mouse Model}

Early dying-back of axons is a hallmark of motor neuron diseases that precedes symptom onset and motor neuron death both in human patients and animal models [28, 31, 44]. In a previous study, we determined that the progression of axonal degeneration in the SOD $1^{\mathrm{G} 93 \mathrm{~A}}$ mouse model of ALS [31] begins after 1 month of age, as mice display no significant degeneration of the LTN at this time. Based on this, we began to treat 1-month-old mice continuously with either vehicle or BACE1 inhibitor orally for 1 month before performing electrophysiological recordings of the CMM followed by the collection of the CMM for morphological analysis.

At the caudal region, where the longest axons reach, vehicle-treated mice had fewer innervated NMJs than the inhibitor-treated mice (Fig. 4A, B). We quantified the percentage of innervated NMJs and determined that inhibitor-treated mice had significantly more innervated NMJs than mice treated with vehicle (Fig. $4 \mathrm{C}$; vehicle $=56.40 \pm 0.38 \%, n=3$; inhibitor $=87.27 \pm 0.80 \%, \mathrm{n}=3 ; p=3.98 \mathrm{E}-6)$. Inhibitor treated mice had overall fewer innervated NMJs than WT mice (Fig. $4 \mathrm{C}$; inhibitor $=87.27 \pm 0.80 \%$; WT $=100 \%, \mathrm{n}=3 ; p=8.96 \mathrm{E}-$ 5 ). At the medial region, we did not observe any significant difference between the vehicle and inhibitor-treated mice, most likely due to there being only minimal degeneration present (Fig. 4C; vehicle $=92.52 \pm 3.23 \%$; inhibitor $=96.19$ 



C

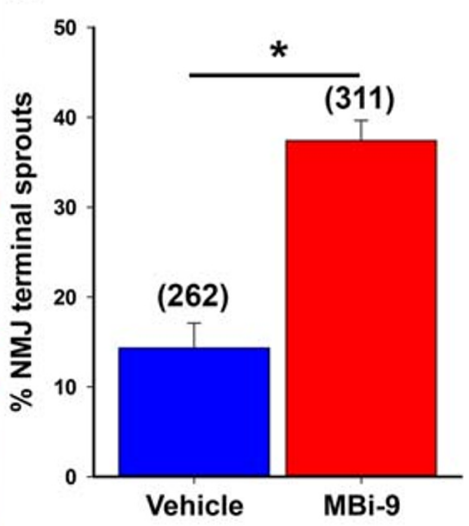

Fig. 3 MBi-9 enhances terminal axonal sprouting following a partial nerve injury. (A, B) Representative images of a mouse treated with vehicle (A) or inhibitor (B) for 2 weeks following a partial LTN transection. White arrows indicate representative terminal axonal sprouts. Inset shows magnified YFP channel of 2 terminal axonal sprouts. (C) Quantification of the percentage of NMJs which have a terminal axonal sprout extending out from itself. Scale bars, $50 \mu \mathrm{m}(\mathbf{A}$, B). Vehicle $n=3$ and inhibitor $n=4$. Numbers in parenthesis represent the total number of NMJs counted. $* p<0.05$. Statistical significance was calculated using a two-tailed Student's $t$ test. Bars represent mean \pm standard error

(vehicle 43.89 $\pm 5.23 \%, n=6$; inhibitor $=69.25 \pm 6.14 \%, n=7$; $p=0.010$ ), and rostral regions (vehicle $62.28 \pm 6.71 \%, n=6$; inhibitor $=83.41 \pm 2.83 \%, n=7 ; p=0.011$ ). Despite the increased innervation with BACE inhibitor treatment, disease progression appears to have continued with lower innervation percentages compared to the inhibitor-treated values after 1 month of treatment (caudal, 1 month $=87.27 \pm 0.80 \%, 2$ months $=53.45 \pm$ $4.82 \%, p=0.0022$; medial, 1 month $=96.19 \pm 2.52 \%$, 2 months $=69.25 \pm 6.14 \%, p=0.025)$. Further progression of the disease is also reflected in the electrophysiological profile of the CMAP amplitudes after 2 months of treatment (Fig. 5D F). While the caudal region showed a significant increase in CMAP amplitudes following 1 month of treatment, after 2 months of treatment we only observed a very weak trend at both the caudal (Fig. 5D; vehicle $0.40 \pm 0.035 \mathrm{mV}, n=34$; inhibitor $=0.46 \pm 0.053 \mathrm{mV}, n=36 ; p=0.67$ ) and medial (Fig. 5E; vehicle $1.84 \pm 0.20 \mathrm{mV}, n=34$; inhibitor $=2.12 \pm 0.22 \mathrm{mV}, n=$ $36 ; p=0.34$ ) region of the CMM. At the rostral region, there was virtually no difference between the inhibitor and vehicle treated mice (Fig. 5F; vehicle $6.71 \pm 0.55 \mathrm{mV}, n=34$; inhibitor $=6.59 \pm$ $0.51 \mathrm{mV}, n=36 ; p=0.93)$. We hypothesize that this loss of significance following 2 months of treatment may be due to irreversible disease progression in SOD $1^{\mathrm{G} 93 \mathrm{~A}}$ that overwhelms the repair process.

\section{Axonal Sprouting Is Enhanced by Reducing BACE Activity}

To determine whether the increased innervation following BACE inhibition in the SOD1 mouse was due to a neuroprotective effect or increased re-innervation, we investigated the extent of terminal axonal sprouting. As determined $n=6$; inhibitor $=53.45 \pm 4.82 \%, n=7 ; p=0.048)$, medial 

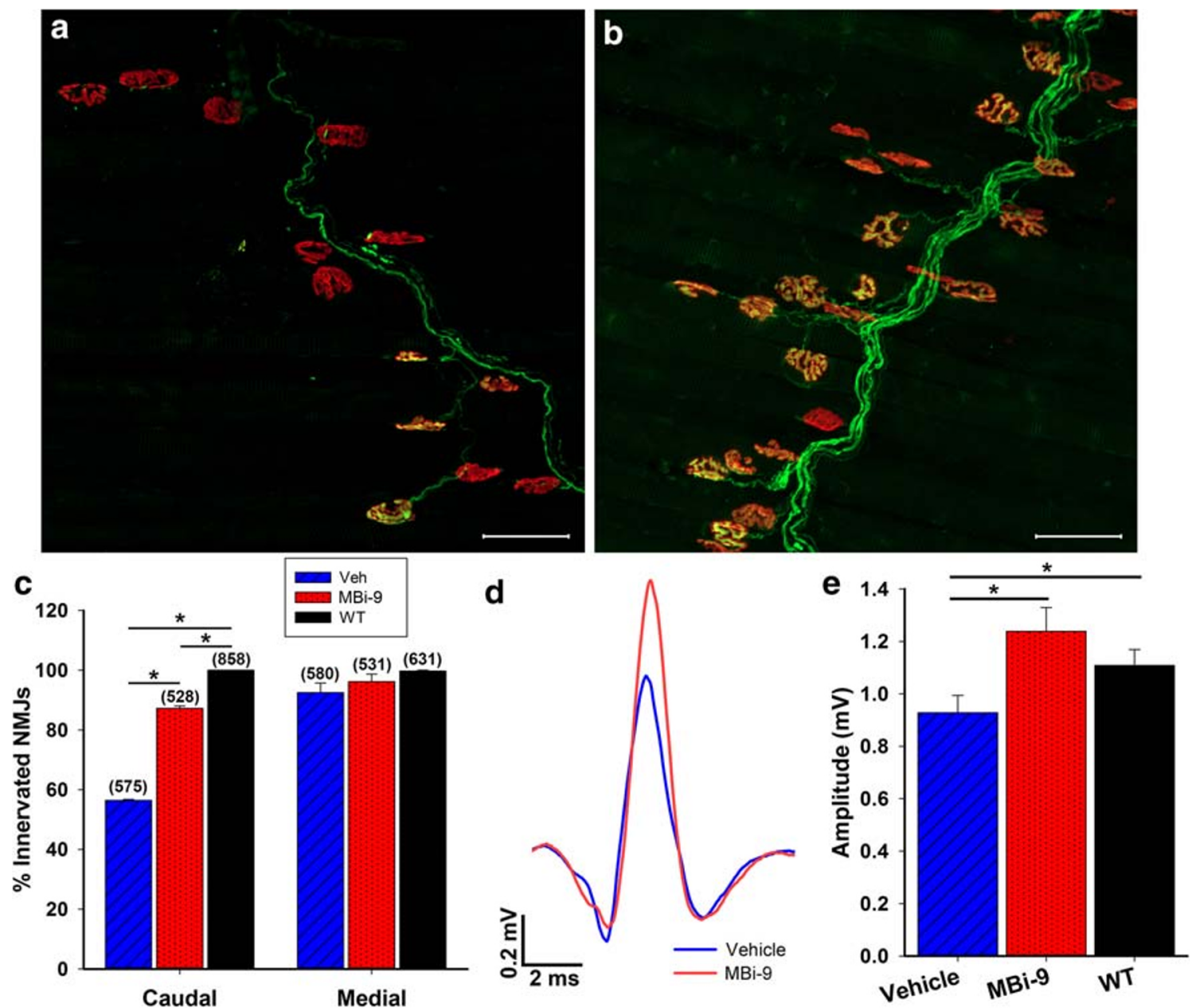

Fig. 41 month of MBi-9 treatment improves morphological and physiological repair in SOD1 mice. (A, B) Representative images of the CMM from the caudal section of a mouse treated for 1 month with vehicle (A) and MBi-9 (B). Axons labeled green and NMJs labeled with red $\alpha$-BTX. (C) Quantification after 1 month of treatment of the percentage of NMJs that are fully innervated in uninjured WT (black, $n=3$ ), vehicle treated SOD1 (blue striped, $n=3$ ), and inhibitor treated SOD1 mice (red dotted, $n=3$ ). Numbers in parenthesis represent total number of NMJs counted.

previously, [31] the extent of axonal sprouting in the SOD1 mouse at 60 days of age was minimal but increased at 90 days. Figure $6 \mathrm{~A}$ and $\mathrm{B}$ show representative caudal images from mice treated for 1 month with either vehicle or MBi-9, respectively. There were many more visible terminal axonal sprouts in the inhibitor-treated mice compared to vehicle-treated mice, as indicated by the white arrows. Following quantification, a significant increase in the percentage of NMJs with the presence of one or more terminal axonal sprouts was observed in inhibitor-treated mice (Fig. 6C; vehicle $4.35 \pm 0.85 \%, n=4$; inhibitor $=12.34 \pm 2.30 \%, n=4 ; p=0.017)$. We continued to observe an increase in the extent of terminal sprouting in the mice treated for 2 months with inhibitor (Fig. 6E) compared to vehicle-treated mice (Fig. 6D) which is quantified in Fig. 6F (vehicle $3.52 \pm 0.99 \%, n=6$; inhibitor $=9.29 \pm 1.98 \%, n=6$; $p=0.026$ ). As was the case with the partial nerve injury, the
All $n=3$. (D) Representative traces of CMAP recordings at the caudal site from vehicle treated SOD1 mice (blue) and inhibitor treated SOD1 mice (red). (E) Quantification of the average CMAPs recorded from WT mice (black), vehicle treated SOD1 mice (blue striped), and inhibitor treated SOD1 mice (red dotted). Scale bars, $50 \mu \mathrm{m}(\mathbf{A}, \mathbf{B})$ and $2 \mathrm{~ms}(x)$ by $0.2 \mathrm{mV}$ (y) (D). WT $n=25$, vehicle $n=23$, and inhibitor $n=23$. Bars represent mean \pm standard error. Statistical significance was calculated using a twotailed student's t test. $* p<0.05$

sprouting percentages in the SOD1 muscles were much higher than those observed in inhibitor treated, uninjured muscles ( $<$ $0.5 \%$, Supplemental figure 4). Based on this finding of increased terminal axonal sprouting following BACE1 inhibitor treatment, we believe that the increased innervation is likely due to enhanced axonal re-innervation as opposed to a neuroprotective effect; however, we cannot exclude the possibility that BACE1 inhibition may also improve the health of intact axons.

\section{CHL1 Expression Levels Are Increased in SOD1 ${ }^{\text {G93A }}$ Mice Following BACE Inhibition}

After determining that BACE1 inhibitors increase NMJ innervation in the early stages of disease in the SOD1 mouse via enhanced axonal sprouting, we next explored a 
Fig. 5 2-month MBIi-9 treatment improves morphological repair but not functional repair. (A, B) Representative images of the

CMM from the caudal section of a mouse treated for 2 months with vehicle (A) and MBI-9 (B).

Axons labeled green and NMJs labeled with red $\alpha$-BTX. (C) Quantification after 2 months of treatment of the percentage of NMJs that are fully innervated in uninjured WT (black, $n=3$ ), vehicle treated SOD1 (blue striped, $n=6$ ), and inhibitor treated SOD1 mice (red dotted, $n=7$ ). Scale bars, $100 \mu \mathrm{m}(\mathbf{A}, \mathbf{B})$. Numbers in parenthesis represent total number of NMJs counted. (D-F) Quantification of the CMAP recordings in vehicle treated SOD1 (blue striped, $n=34$ ), and inhibitor treated SOD1 mice (red dotted, $n=36$ ) at the caudal (D), medial $(\mathbf{E})$, and rostral sites $(\mathbf{F})$. Bars represent mean \pm standard error. Statistical significance was calculated using a two-tailed Student's $t$ test (C) or MannWhitney's rank sum test (D-F). $* p<0.05$
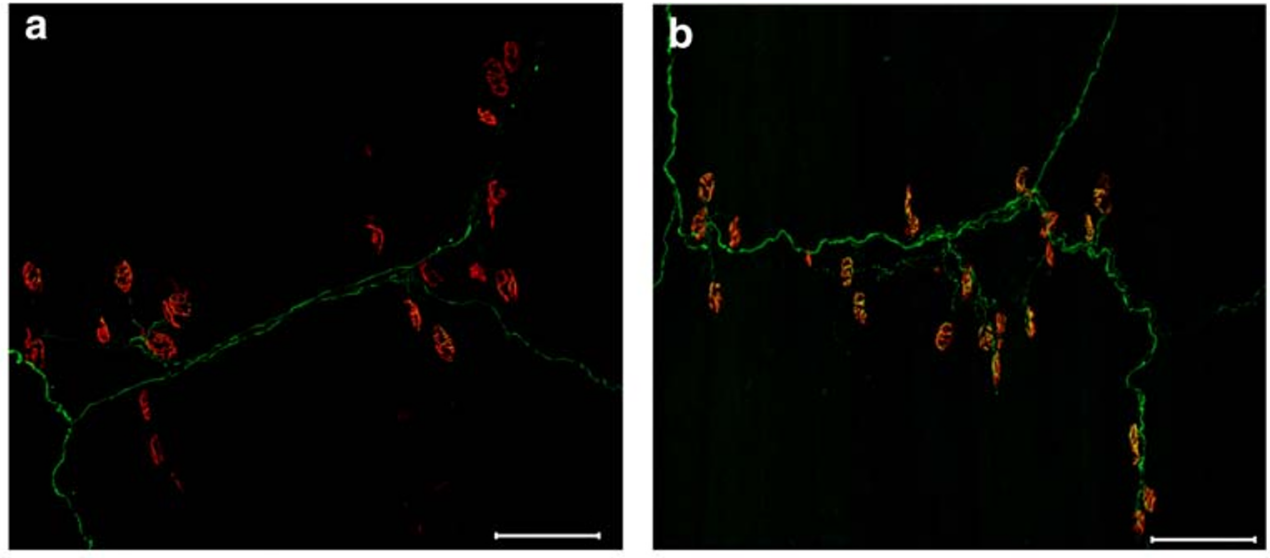

c
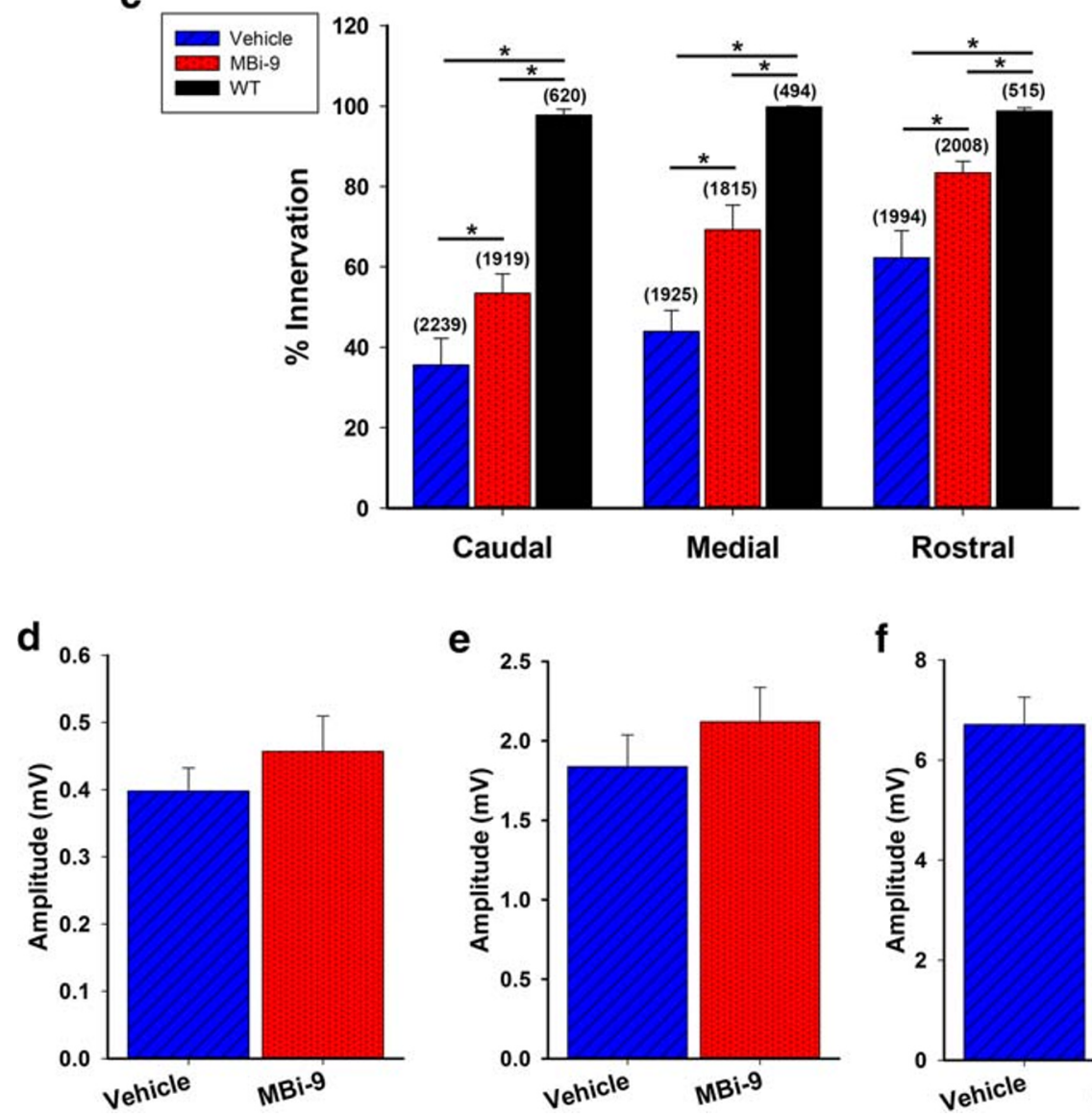

e

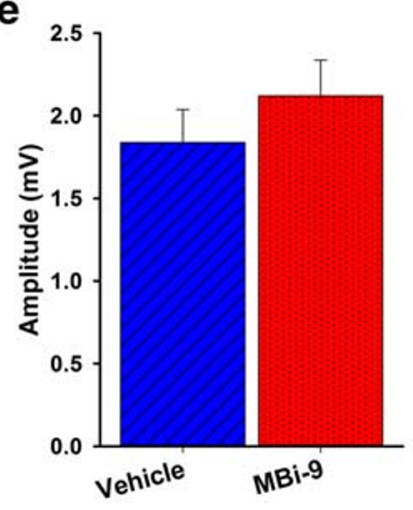

f

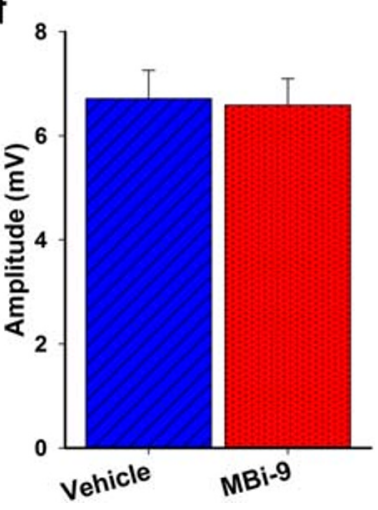

potential molecular mechanism. Since BACE1 cleaves numerous single transmembrane proteins, [5] we decided to narrow our initial investigation to CHL1, a validated BACE1 substrate known to be involved in regulating axon biology, $[18,19]$ in order for axons to sprout and be guided towards a target they need to interact with the supporting Schwann cell tubes. We therefore hypothesized that BACE1 may be modulating expression levels of neuronal adhesion molecules as a way of controlling this axonSchwann cell interaction. We decided to investigate whether the levels of full-length CHL1 were altered in SOD1 mice treated with MBi-9. Western blot analysis of spinal cord extracts for CHL1 levels indicated a significant increase in the normalized intensity of CHL1 in the inhibitor-treated mice compared with the vehicle-treated animals (Fig. 7A, B; vehicle $=0.87 \pm 0.042, n=4$; inhibitor $=1.03 \pm 0.22, n=4 ; p=0.015$ ). This result supports our hypothesis that BACE1 activity levels modulate expression levels of full-length adhesion molecules that have been shown to regulate axonal outgrowth. 

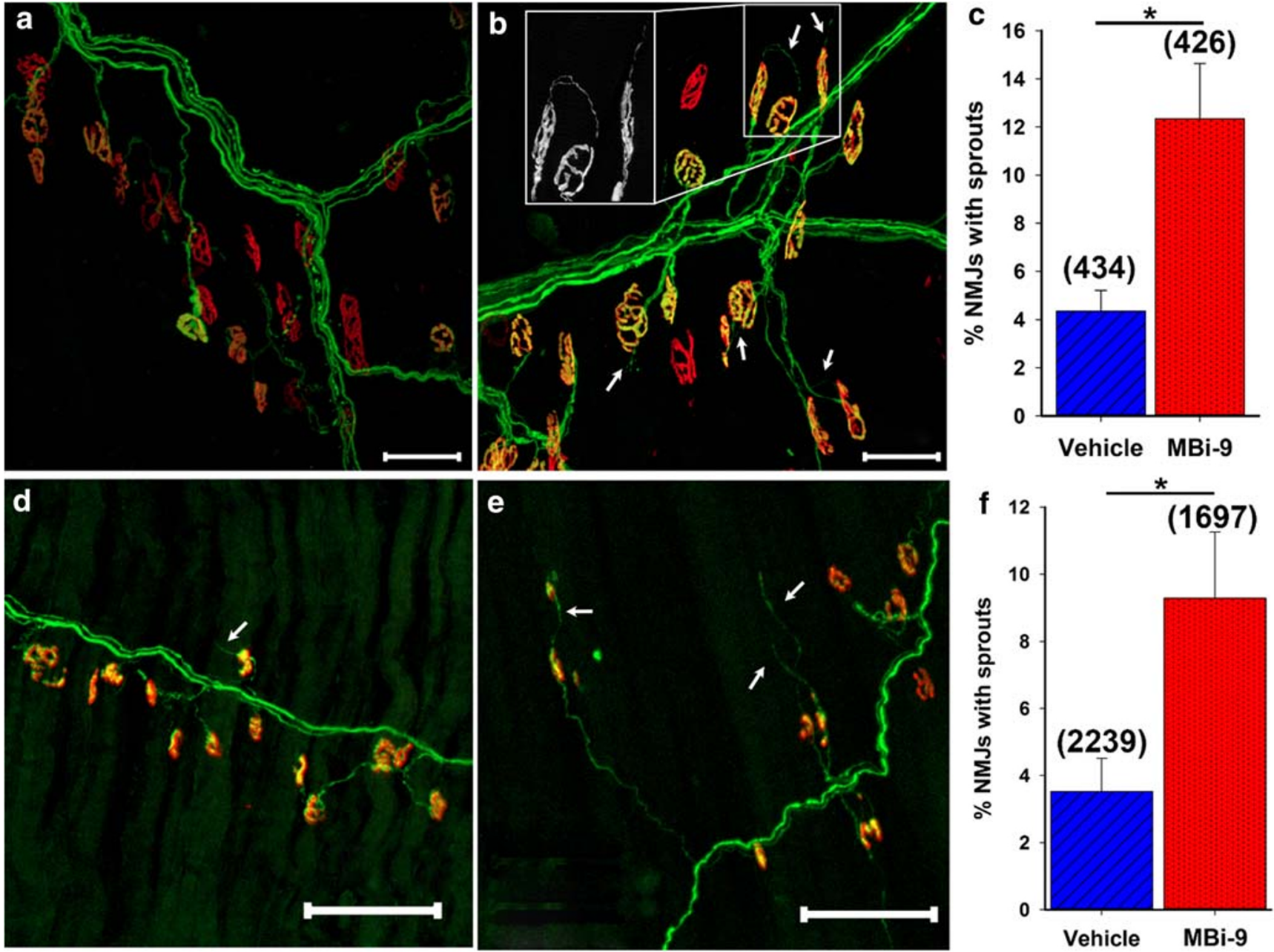

Fig. 6 BACE inhibition enhances axonal sprouting in SOD1 mice. (A, B) Representative sprouting images of 1-month-old SOD1 mice treated with vehicle (D) or MBi-9 (E). (F) Quantification of the percentage of NMJs with terminal sprouts following 2 months of treatment. $N=6$ mice each. Numbers in parenthesis represent total number of NMJs counted. Bars represent mean \pm standard error. Scale bars $50 \mu \mathrm{m}(\mathbf{A}, \mathbf{B})$ and $100 \mu \mathrm{m}(\mathbf{D}$, NMJs are labeled red. White arrows point to terminal axonal sprouts. Inset shows magnified terminal axonal sprout. (C) Quantification of the percentage of NMJs with terminal sprouts after 1 month of treatment. $n=$ 4 each. (D, E) Representative images of mice treated for 2 months with

E). Statistical significance was calculated using a two-tailed Student's $t$ test. $* p<0.05$

Fig. 7 BACE inhibition alters CHL1 expression levels in SOD1 mice. (A) Representative Western blot of spinal cord tissue collected from SOD $1^{\mathrm{G} 93 \mathrm{~A}}$ mice following 1 month of either vehicle or BACE inhibitor treatment. GAPDH was used as a loading control for normalization calculations. (B) Quantification of the Western blots following normalization of the CHL1 band intensity to the GAPDH band intensity. Bars represent mean \pm standard error. Statistical significance was calculated using a two-tailed Student's $t$ test. ${ }^{*} p<0.05$ a

\section{Vehicle MBi-9}

CHL1

\section{GAPDH}

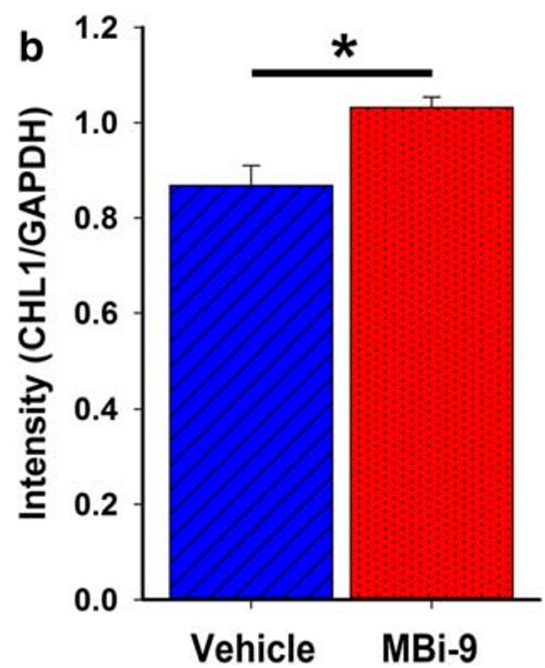




\section{Discussion}

The data presented here strongly demonstrated that BACE inhibition enhanced peripheral nerve regeneration following a sciatic nerve crush injury as well as improved muscle innervation in the SOD $1^{\mathrm{G} 93 \mathrm{~A}}$ mouse model of ALS, indicating that BACE inhibition may be an avenue for improving functional output following a traumatic nerve injury as well as in the early stages of motor neuron diseases.

In this report, we demonstrated that pharmacologic inhibition of BACE enhanced peripheral nerve regeneration after a sciatic nerve injury. Recently, well-characterized and clinically well-tolerated small molecule inhibitors $[22,23]$ have been developed for BACE1 due to its role in the proteolytic cleavage of amyloid precursor protein which has been implicated in Alzheimer's Disease [6-8]. We demonstrated that mice treated with a BACE inhibitor had significantly higher numbers of regenerated axons and innervated NMJs 2 weeks after sciatic nerve crush. Another important finding was that myelination of uninjured nerves remained unchanged in uninjured WT mice treated with the BACE inhibitor. This indicates that BACE inhibitor treatment did not negatively affect normal nerve morphology and would therefore not be likely to cause any unintentional decline in normal motor and sensory function. These results support the idea that pharmacologic attenuation of BACE1 activity could be a potential clinical intervention for peripheral nerve repair.

Interestingly, we observed that short-term BACE inhibition was sufficient to improve functional recovery after nerve injury. We observed that despite having similar amplitudes, the area under the curve of the CMAP traces following a sciatic nerve crush injury was greater in treated mice with a longer latency, suggesting that while there were more axons in BACE inhibitor-treated nerves, they were firing asynchronously. We hypothesized that this difference was due to reduced axonal re-myelination in BACE inhibitor treated nerves due to the inhibition of neuregulin processing by BACE1. [9, 10, 42, 43] To address this point, we chose a short treatment duration, which leads to long-term enhanced functional recovery. After a 2-week washout of the inhibitor, we found that the previously treated axons were now able to fire together and generate significantly greater amplitudes after only 2 weeks without inhibitor. Additionally, it would be beneficial to expand on this study and carry out extensive dose-response experiments to identify the minimum effective dose. It is possible that a lower effective dose may have a milder impact on remyelination which would allow for increased therapeutic treatment windows.

Using a partial nerve injury model, we examined collateral sprouting, where intact axons sprout towards denervated muscle in order to re-innervate vacant NMJs. Most traumatic nerve injuries are partial injuries that leave some axons intact
[45-47]. In non-pathologic conditions, one axon terminal innervates one NMJ; however, when there is partial denervation due to injury or disease, one axon terminal is capable of innervating up to $5 \mathrm{NMJs}$, and this translates to some retention of motor function [48]. This is important for transections of very long axons as the rate of regeneration is incredibly slow, around $1 \mathrm{~mm}$ per day, $[49,50]$ and collateral sprouting can reinnervate the muscle before any degeneration of the muscle occurs due to a lack of any neuronal input [51]. The data presented here demonstrated that mice treated with MBi-9 had significantly more terminal axonal sprouts than vehicletreated mice suggesting that BACE inhibition encourages outgrowth of severed axons and sprouting of intact axons to reach vacant NMJs.

We also explored whether BACE inhibitors could improve re-innervation in the SOD1 mouse model of ALS. By promoting the plasticity of NMJs, the progression of muscle weakness could be delayed, potentially enhancing patient quality-of-life. SOD1 ${ }^{\mathrm{G} 93 \mathrm{~A}}$ mice exhibit denervation of NMJs before motor neuron cell death, providing time for axonal sprouting and NMJ re-innervation to occur. We demonstrated that BACE inhibition improved morphological and functional recovery following 1 month of treatment, however, after 2 months of treatment, only morphological re-innervation was enhanced with only a trend in functional recovery. This may be due to the advancement of the disease affecting the overall health of the motor neuron causing substantial death to the point where it overwhelms the repair process. [48] Another possibility could be that the size of the motor units has become too large for the neurons to be able to support extensive neuromuscular connections and therefore leads to an impaired firing capacity. We also observed enhanced terminal axonal sprouting in SOD1 ${ }^{\mathrm{G} 93 \mathrm{~A}}$ mice following BACE inhibitor treatment further supporting the hypothesis that the improved innervation seen with BACE inhibition was due to enhanced re-innervation and not a neuroprotective effect [52].

Importantly, we did not observe any increase in terminal sprouting in WT mice treated with our BACE inhibitor. It has been demonstrated that blocking synaptic transmission at the NMJ either by using tetrodotoxin or botulinum toxin leads to compensatory sprouting without the need for axonal degeneration $[40,41]$. That we did not observe any increase in terminal sprouts following BACE inhibitor treatment suggests that the increase in axonal sprouting reported in this manuscript is not working through a similar compensatory sprouting mechanism due to transmission blockage but more likely due to an enhancement of the intrinsic repair mechanisms in response to axonal injury.

A number of experimental interventions have been reported to increase motor axon sprouting. Administration of exogenous neuregulin, ATF3 overexpression and targeted 
deletion of APP have demonstrated increased motor axon sprouting, improved motor function and muscle force, and delayed disease onset in SOD1 mice [53-55]. ATF3 overexpression, APP deletion, and neuregulin administration also have effects on neuroprotection in SOD1 mice [53, $54,56]$. The degree to which increased motor axon sprouting versus neuroprotection contributes to improved disease outcomes is unknown, however, it was recently shown that compensatory sprouting was able to fully maintain muscle function in a transgenic mouse model of spinal muscular atrophy type III [57]. Enhancing the compensatory efforts of motor axons to reinnervate NMJs is a promising approach to slow symptom progression in motor neuron disease, but transgenic approaches are not easily clinically translatable. Utilizing a small molecule inhibitor to increase motor axon sprouting, such as BACE inhibitors, would be a preferable therapeutic candidate. In support of this notion, a small molecule inhibitor of RhoA, Y-27632, has led to enhanced regeneration of motor axons after a sciatic nerve crush and increased reinnervation in the SOD1 mice $[58,59]$. Our data also points to the feasibility of small molecule inhibitors as a novel approach to enhancing sprouting and improving reinnervation in both nerve injury and motor neuron disease.

In addition to identifying BACE inhibitors as potential therapeutics, we also began to explore potential mechanisms underlying this enhanced regeneration. Previously, we demonstrated that neuronal BACE1 overexpression can sufficiently impair regeneration without any altered BACE1 expression levels in the Schwann cells or macrophages [17]. Since Schwann cell-axon interactions are extremely important for proper axonal regeneration, we hypothesized that BACE1 mediated cleavage of cell adhesion molecules could potentially be the mechanism by which BACE1 is able to regulate peripheral nerve regeneration. Indeed, BACE1 is involved in the cleavage of L1 and CHL1 [12, 21], 2 cell adhesion molecules, which have been implicated in regulating axonal outgrowth via interactions with Schwann cells in vitro [18, 19, 60, 61]. We were able to observe an increase in the expression levels of full-length CHL1 in inhibitor-treated mice compared to those given the vehicle treatment. These findings point towards the possibility that reduced cleavage of CHL1 by BACE1 may increase the amount of full-length CHL1 available at the membrane which could enhance Schwann cell-axon interactions leading to improved regeneration.

By demonstrating improved regrowth and sprouting in response to both a crush and partial transection injury, BACE inhibition has the potential to improve regeneration following a wide variety of nerve injury scenarios. With regard to motor neuron disease and chronic peripheral neuropathies, our data supports early intervention in order to improve muscle innervation, which limits the treatment groups to those with early signs of motor neuron disease before overt clinical symptoms develop. Despite these limitations, we believe that our study provides a promising first step towards utilizing BACE inhibitors as a therapy for enhancing peripheral nerve regeneration following injury and disease.

Acknowledgments The authors thank Katelyn Russell, Lijuan Liu, Ping Xue, Ashley Lea, and Aniqa Tasnim for technical assistance. We also thank Carol Cooke for electron microscopy assistance.

Required Author Forms Disclosure forms provided by the authors are available with the online version of this article.

Author Contributions C.T., K.L.M., and M.H.F. designed and performed in vivo and in vitro experiments. M.E.K. provided the MBi-9 inhibitor. C.T. and M.H.F. wrote the paper with input from all the listed authors.

Funding This work was supported by the Muscular Dystrophy Association, W81XWH1910229 from Department of Defense's congressionally directed medical research program, and R01NS079339 from the National Institutes of Neurological Disease and Stroke of the National Institutes of Health. This work was also partially supported by Merck \& Co. Inc.

\section{References}

1. Gordon T. Nerve regeneration in the peripheral and central nervous systems. J Physiol, 594(13), 3517-3520 (2016).

2. Scheib J, Hoke A. Advances in peripheral nerve regeneration. Nat Rev Neurol, 9(12), 668-676 (2013).

3. Griffin JW, Pan B, Polley MA, Hoffman PN, Farah MH. Measuring nerve regeneration in the mouse. Exp Neurol, 223(1), 60-71 (2010).

4. Palispis WA, Gupta R. Surgical repair in humans after traumatic nerve injury provides limited functional neural regeneration in adults. Exp Neurol, 290, 106-114 (2017).

5. Hemming ML, Elias JE, Gygi SP, Selkoe DJ. Identification of betasecretase (BACE1) substrates using quantitative proteomics. PLoS One, 4(12), e8477 (2009).

6. Sinha S, Anderson JP, Barbour R et al. Purification and cloning of amyloid precursor protein beta-secretase from human brain. Nature, 402(6761), 537-540 (1999).

7. Vassar R, Bennett BD, Babu-Khan S et al. Beta-secretase cleavage of Alzheimer's amyloid precursor protein by the transmembrane aspartic protease BACE. Science, 286(5440), 735-741 (1999).

8. Yan R, Bienkowski MJ, Shuck ME et al. Membrane-anchored aspartyl protease with Alzheimer's disease beta-secretase activity. Nature, 402(6761), 533-537 (1999).

9. Hu X, Hicks CW, He W et al. Bacel modulates myelination in the central and peripheral nervous system. Nat Neurosci, 9(12), 1520 1525 (2006).

10. Willem M, Garratt AN, Novak B et al. Control of peripheral nerve myelination by the beta-secretase BACE1. Science, 314(5799), 664-666 (2006).

11. Kuhn PH, Marjaux E, Imhof A, De Strooper B, Haass C, Lichtenthaler SF. Regulated intramembrane proteolysis of the interleukin-1 receptor II by alpha-, beta-, and gamma-secretase. $J$ Biol Chem, 282(16), 11982-11995 (2007).

12. Zhou L, Barão $\mathrm{S}$, Laga $\mathrm{M}$ et al. The neural cell adhesion molecules $\mathrm{L} 1$ and $\mathrm{CHL} 1$ are cleaved by BACE1 protease in vivo. $J$ Biol Chem, 287(31), 25927-25940 (2012).

13. Pigoni M, Wanngren J, Kuhn PH et al. Seizure protein 6 and its homolog seizure 6-like protein are physiological substrates of BACE1 in neurons. Mol Neurodegener, 11(1), 67 (2016). 
14. Michailov GV, Sereda MW, Brinkmann BG et al. Axonal neuregulin-1 regulates myelin sheath thickness. Science, 304(5671), 700-703 (2004).

15. Taveggia C, Zanazzi G, Petrylak A et al. Neuregulin-1 type III determines the ensheathment fate of axons. Neuron, 47(5), 681694 (2005).

16. Farah MH, Pan BH, Hoffman PN et al. Reduced BACE1 activity enhances clearance of myelin debris and regeneration of axons in the injured peripheral nervous system. $J$ Neurosci, 31(15), 5744 5754 (2011).

17. Tallon C, Rockenstein E, Masliah E, Farah MH. Increased BACE1 activity inhibits peripheral nerve regeneration after injury. Neurobiol Dis, 106, 147-157 (2017).

18. Chaisuksunt V, Campbell G, Zhang Y, Schachner M, Lieberman $\mathrm{AR}$, Anderson PN. The cell recognition molecule CHL1 is strongly upregulated by injured and regenerating thalamic neurons. J Comp Neurol, 425(3), 382-392 (2000).

19. Zhang Y, Roslan R, Lang D, Schachner M, Lieberman AR, Anderson PN. Expression of CHL1 and L1 by neurons and glia following sciatic nerve and dorsal root injury. Mol Cell Neurosci, 16(1), 71-86 (2000).

20. Hinkle CL, Diestel S, Lieberman J, Maness PF. Metalloproteaseinduced ectodomain shedding of neural cell adhesion molecule (NCAM). J Neurobiol, 66(12), 1378-1395 (2006).

21. Barão S, Gärtner A, Leyva-Díaz E et al. Antagonistic Effects of BACE1 and APH1B- $\gamma$-Secretase Control Axonal Guidance by Regulating Growth Cone Collapse. Cell Rep, 12(9), 1367-1376 (2015).

22. Kennedy ME, Stamford AW, Chen X et al. The BACE1 inhibitor verubecestat (MK-8931) reduces CNS $\beta$-amyloid in animal models and in Alzheimer's disease patients. Sci Transl Med, 8(363), 363ra150 (2016).

23. Egan MF, Kost J, Voss Tet al. Randomized Trial of Verubecestat for Prodromal Alzheimer's Disease. N Engl J Med, 380(15), 14081420 (2019).

24. Pan B, Grunewald B, Nguyen T et al. The lateral thoracic nerve and the cutaneous maximus muscle-a novel in vivo model system for nerve degeneration and regeneration studies. Exp Neurol, 236(1), 6-18 (2012).

25. Hegedus J, Putman CT, Gordon T. Time course of preferential motor unit loss in the SOD1 G93A mouse model of amyotrophic lateral sclerosis. Neurobiol Dis, 28(2), 154-164 (2007).

26. Pun S, Santos AF, Saxena S, Xu L, Caroni P. Selective vulnerability and pruning of phasic motoneuron axons in motoneuron disease alleviated by CNTF. Nat Neurosci, 9(3), 408-419 (2006).

27. Schaefer AM, Sanes JR, Lichtman JW. A compensatory subpopulation of motor neurons in a mouse model of amyotrophic lateral sclerosis. J Comp Neurol, 490(3), 209-219 (2005).

28. Fischer LR, Culver DG, Tennant $\mathrm{P}$ et al. Amyotrophic lateral sclerosis is a distal axonopathy: evidence in mice and man. Exp Neurol, 185(2), 232-240 (2004).

29. Frey D, Schneider C, Xu L, Borg J, Spooren W, Caroni P. Early and selective loss of neuromuscular synapse subtypes with low sprouting competence in motoneuron diseases. J Neurosci, 20(7), 2534-2542 (2000).

30. Azzouz M, Leclerc N, Gurney M, Warter JM, Poindron P, Borg J. Progressive motor neuron impairment in an animal model of familial amyotrophic lateral sclerosis. Muscle Nerve, 20(1), 45-51 (1997).

31. Tallon C, Russell KA, Sakhalkar S, Andrapallayal N, Farah MH. Length-dependent axo-terminal degeneration at the neuromuscular synapses of type II muscle in SOD1 mice. Neuroscience, 312, 179189 (2016).

32. Bame M, Pentiak PA, Needleman R, Brusilow WSA. Effect of Sex on Lifespan, Disease Progression, and the Response to Methionine
Sulfoximine in the SOD1 G93A Mouse Model for ALS. Gender Medicine, 9(6), 524-535 (2012).

33. May PC, Willis BA, Lowe SL et al. The potent BACE1 inhibitor LY2886721 elicits robust central Abeta pharmacodynamic responses in mice, dogs, and humans. J Neurosci, 35(3), 1199-1210 (2015).

34. Stamford AW, Scott JD, Li SW et al. Discovery of an Orally Available, Brain Penetrant BACE1 Inhibitor that Affords Robust CNS A $\beta$ Reduction. ACS Med Chem Lett, 3(11), 897-902 (2012).

35. Mandal M, Wu Y, Misiaszek J et al. Structure-Based Design of an Iminoheterocyclic $\beta$-Site Amyloid Precursor Protein Cleaving Enzyme (BACE) Inhibitor that Lowers Central $A \beta$ in Nonhuman Primates. Journal of Medicinal Chemistry, 59(7), 3231-3248 (2016).

36. Feng G, Mellor RH, Bernstein M et al. Imaging neuronal subsets in transgenic mice expressing multiple spectral variants of GFP. Neuron, 28(1), 41-51 (2000).

37. Gurney $\mathrm{ME}, \mathrm{Pu} \mathrm{H}$, Chiu AY et al. Motor neuron degeneration in mice that express a human $\mathrm{Cu}, \mathrm{Zn}$ superoxide dismutase mutation. Science, 264(5166), 1772-1775 (1994).

38. Cai H, Wang Y, McCarthy D et al. BACE1 is the major betasecretase for generation of Abeta peptides by neurons. Nat Neurosci, 4(3), 233-234 (2001).

39. Scott JD, Li SW, Brunskill AP et al. Discovery of the 3-Imino-1,2, 4-thiadiazinane 1,1-Dioxide Derivative Verubecestat (MK-8931)-A beta-Site Amyloid Precursor Protein Cleaving Enzyme 1 Inhibitor for the Treatment of Alzheimer's Disease. J Med Chem, 59(23), 10435-10450 (2016)

40. Brown MC, Ironton R. Motor neurone sprouting induced by prolonged tetrodotoxin block of nerve action potentials. Nature, 265(5593), 459-460 (1977).

41. Pestronk A, Drachman D. Motor nerve sprouting and acetylcholine receptors. Science, 199(4334), 1223-1225 (1978).

42. $\mathrm{Hu} \mathrm{X}, \mathrm{He} \mathrm{W}$, Diaconu $\mathrm{C}$ et al. Genetic deletion of BACE1 in mice affects remyelination of sciatic nerves. Faseb j, 22(8), 2970-2980 (2008).

43. $\mathrm{Hu} \mathrm{X}, \mathrm{Hu}$ J, Dai L, Trapp B, Yan R. Axonal and Schwann cell BACE1 is equally required for remyelination of peripheral nerves. J Neurosci, 35(9), 3806-3814 (2015).

44. Dadon-Nachum M, Melamed E, Offen D. The "dying-back" phenomenon of motor neurons in ALS. J Mol Neurosci, 43(3), 470 477 (2011).

45. Eggers R, Tannemaat MR, De Winter F, Malessy MJ, Verhaagen J. Clinical and neurobiological advances in promoting regeneration of the ventral root avulsion lesion. Eur J Neurosci, 43(3), 318-335 (2016).

46. Gordon T, Amirjani N, Edwards DC, Chan KM. Brief post-surgical electrical stimulation accelerates axon regeneration and muscle reinnervation without affecting the functional measures in carpal tunnel syndrome patients. Exp Neurol, 223(1), 192-202 (2010).

47. Gordon T, Borschel GH. The use of the rat as a model for studying peripheral nerve regeneration and sprouting after complete and partial nerve injuries. Exp Neurol, 287(Pt 3), 331-347 (2017).

48. Gordon T, Hegedus J, Tam SL. Adaptive and maladaptive motor axonal sprouting in aging and motoneuron disease. Neurol Res, 26(2), 174-185 (2004)

49. Hoke A. Neuroprotection in the peripheral nervous system: rationale for more effective therapies. Arch Neurol, 63(12), 1681-1685 (2006).

50. Gordon T, Tyreman N, Raji MA. The basis for diminished functional recovery after delayed peripheral nerve repair. $J$ Neurosci, 31(14), 5325-5334 (2011).

51. Sakuma M, Gorski G, Sheu S-H et al. Lack of motor recovery after prolonged denervation of the neuromuscular junction is not due to regenerative failure. European Journal of Neuroscience, 43(3), 451-462 (2016). 
52. Nikolaev A, McLaughlin T, O'Leary DD, Tessier-Lavigne M. APP binds DR6 to trigger axon pruning and neuron death via distinct caspases. Nature, 457(7232), 981-989 (2009).

53. Bryson JB, Hobbs C, Parsons MJ et al. Amyloid precursor protein (APP) contributes to pathology in the SOD1(G93A) mouse model of amyotrophic lateral sclerosis. Hum Mol Genet, 21(17), 38713882 (2012).

54. Seijffers R, Zhang J, Matthews JC et al. ATF3 expression improves motor function in the ALS mouse model by promoting motor neuron survival and retaining muscle innervation. Proc Natl Acad Sci U $S$ A, 111(4), 1622-1627 (2014).

55. Mancuso R, Martinez-Muriana A, Leiva T et al. Neuregulin-1 promotes functional improvement by enhancing collateral sprouting in SOD1(G93A) ALS mice and after partial muscle denervation. Neurobiol Dis, 95, 168-178 (2016).

56. Kataria H, Alizadeh A, Karimi-Abdolrezaee S. Neuregulin-1/ErbB network: An emerging modulator of nervous system injury and repair. Prog Neurobiol, 101643 (2019).

57. Udina E, Putman CT, Harris LR, Tyreman N, Cook VE, Gordon T. Compensatory axon sprouting for very slow axonal die-back in a transgenic model of spinal muscular atrophy type III. $J$ Physiol, 595(5), 1815-1829 (2017)

58. Joshi AR, Bobylev I, Zhang G, Sheikh KA, Lehmann HC. Inhibition of Rho-kinase differentially affects axon regeneration of peripheral motor and sensory nerves. Exp Neurol, 263, 28-38 (2015).

59. Joshi AR, Muke I, Bobylev I, Lehmann HC. ROCK inhibition improves axonal regeneration in a preclinical model of amyotrophic lateral sclerosis. J Comp Neurol, (2019).

60. Bixby JL, Lilien J, Reichardt LF. Identification of the major proteins that promote neuronal process outgrowth on Schwann cells in vitro. J Cell Biol, 107(1), 353-361 (1988).

61. Seilheimer B, Schachner M. Studies of adhesion molecules mediating interactions between cells of peripheral nervous system indicate a major role for L1 in mediating sensory neuron growth on Schwann cells in culture. J Cell Biol, 107(1), 341-351 (1988).

Publisher's Note Springer Nature remains neutral with regard to jurisdictional claims in published maps and institutional affiliations. 